\title{
Preschoolers Learn New Moral and Conventional Norms from Direct
}

\section{Experiences}

Word count: 10383 words (text plus references).

Date of submission: September 21, 2021

Declaration of interest: none.

To be published in Journal of Experimental Child Psychology. This is a preprint and may contain errors or omissions not present in the published version. 


\begin{abstract}
By observing others, children can learn about different types of norms, including moral norms rooted in concerns for welfare and rights, and social conventions based on directives from authority figures or social consensus. Two studies examined how preschoolers and adults constructed and applied knowledge about novel moral and conventional norms from their direct social experiences. Participants watched a video of a novel prohibited action that caused pain to a victim (moral conditions) or a sound from a box (conventional conditions), and then saw a transgressor puppet, who had either watched the video alongside the participant or not, engage in the prohibited action. Preschoolers and adults rapidly constructed distinct moral and conventional evaluations about the novel actions. These distinctions were evident across several response modalities that have often been studied separately, including judgments, reasoning, and actions. However, children did not reliably track the puppet's knowledge of the novel norms. These studies provide experimental support for the idea that children and adults construct distinct moral and conventional norms from social experiences, which in turn guide judgments, reasoning, and behavior.
\end{abstract}

Keywords: moral development; conventional development; social domain theory; moral judgment; Theory of Mind 


\section{Preschoolers Learn New Moral and Conventional Norms from Direct Experiences}

Children's understanding and application of different social norms is critical for navigating the social world (Killen \& Smetana, 2015; Tomasello, 2016). For instance, children need to learn that some social norms — like the moral prohibition against hittingapply in nearly all contexts, whereas other norms-including social conventions like dress codes - apply in some contexts and not others. Such distinctions can be learned from direct social experiences (Davidson et al., 1983; Dahl \& Turiel, 2019; Smetana, 2012; Turiel, 1983). A child might observe that hitting others elicits pain signals from a victim, whereas dress code violations merely elicit prohibitions from teachers or parents but do not have inherent consequences. Such observations provide a basis for constructing distinct norms that shape children's judgments, reasoning, and decisions about similar actions in the future (Killen \& Smetana, 2015). They could also allow children to infer whether others are likely to share their knowledge of such norms.

The present experiments investigated the construction of new moral and conventional norms from direct social experiences. Specifically, our studies assessed preschoolers' and adults' normative judgments and decisions in situations where they had not previously been exposed to the norms in question. By moral norms, we mean evaluative principles that protect others' welfare, rights, fairness, and justice, such as prohibitions against hitting or stealing (Turiel, 1983, 2015; Turiel \& Dahl, 2019). By conventional norms, we mean evaluative principles that are rooted in concerns with authorities, consensus, or tradition, such as norms for eating, dressing, or speaking (Dahl \& Waltzer, 2020; Lewis, 1969; Turiel, 2015).

Our studies had three goals. First, we aimed to provide a direct experimental test of how children construct novel moral and conventional norms from social experiences. 
Second, we sought to integrate two segregated methodological approaches to studying children's developing understanding of norms — one studying judgments and reasoning, another studying interventions_-by assessing children's judgments, reasoning, and interventions within the same study. Third, we examined whether children expect other agents to construct novel moral or conventional norms as a function of their own social experiences.

\section{How Do Preschoolers and Adults Use Distinct Social Experiences to Construe New Moral or Conventional Norms?}

Across cultures, children distinguish moral from conventional norms by age three (see Killen \& Smetana, 2015). In interviews about hypothetical transgressions, children commonly reason about moral norms based on concerns with others' welfare, fairness or rights, and about conventional norms based on concerns with authority, rules, consensus, or tradition (Davidson et al., 1983; Nucci \& Turiel, 1978; Smetana, 1985). Children also typically judge moral transgressions to be more severe, more punishable, and more generalizable across contexts than conventional transgressions, and they usually view conventional norms as more alterable (e.g. Nucci \& Nucci, 1982; Smetana, 1981; Smetana \& Braeges, 1990).

But how do children learn to make these distinctions? One hypothesis is that children's construal of a new norm as moral or conventional emerges from their direct social experiences (e.g., Dahl \& Freda, 2017; Smetana, 2013; Turiel, 1983, 2002, 2015). Moral norms involve inherent consequences to victims, such as harm or the violation of rights, while conventional norms do not (Turiel, 1983, 2015). Thus, children might infer a new moral norm directly from observing the reactions of a victim. In contrast, children might learn about conventional norms primarily from authorities, such as teachers or parents, or through references to existing rules (Turiel, 1983, 2015). 
The proposal that children construct the moral/conventional distinction through distinct social experiences has received empirical support from observational studies, which have demonstrated that moral and conventional norm violations are associated with distinct experiences in homes and preschools (e.g. Dahl et al., 2014; Nucci \& Nucci, 1982; Nucci \& Turiel, 1987; Siegal \& Storey, 1985). For instance, parents use more angry vocalizations in response to moral transgressions than other transgressions (Dahl et al., 2014). Moreover, moral violations tend to elicit references to how the actions affected child or adult victims, whereas conventional violations more often elicit references to existing rules from adults (Nucci \& Nucci, 1982; Nucci \& Turiel, 1978; Smetana, 1989; see Smetana, 2013).

While some of the events captured in these observational studies were likely novel to children, others were likely similar to those they had encountered before (e.g., a child hitting another). Because these studies did not separate novel and familiar events in their analyses, they are limited in their ability to reveal children's construction of novel norms in response to novel social events. That is, we cannot know whether children construed novel moral or conventional norms in these situations based on the distinct social experiences associated with them (e.g., that pushing another child off a swing causes harm to a victim, while not sitting down while eating a snack does not). When children in these previous studies encountered familiar events, they could have instead applied norms that they had learned about previously, via socialization from adults, who told them that, e.g., pushing others is wrong because it causes harm (hence a moral violation), while not sitting down while eating a snack is wrong because it violates a school rule (hence a conventional violation; see Kochanska \& Aksan, 2006). Thus, experiments that present children with novel events can provide an important complement to prior observational studies in corroborating Turiel and colleagues' thesis that children construct moral and conventional concepts from their experiences. 
Naturalistic and experimental methods serve complementary roles in developmental science, with both methods having inherent advantages and disadvantages (see Dahl, 2017). An advantage of the experimental design adopted in the present study is that it presented children with novel norms and experimentally manipulated how they were introduced to those norms. Some researchers have presented children with hypothetical stories which varied the typical features of moral and conventional acts (e.g., situations in which an animal experienced pleasure from being hit, and pain from being pet, Zelazo et al., 1996, see also Helwig et al., 2001). In contrast, in the present research, we presented children with live vignettes involving norms that were truly novel: children witnessed live events in which novel actions either elicited both victim suffering and adult prohibition (moral violation) or merely adult prohibition (conventional violation). This experimental paradigm thus offered a crucial examination of the processes by which children construct distinct moral and conventional norms from social experiences.

\section{Do Preschoolers and Adults Show Cognitive and Motivational Elements of Norm Endorsement Within the Same Paradigm?}

Prior research has often assessed the cognitive and motivational components of norm endorsement separately, despite the fact that endorsing a new norm involves both elements (Tomasello, 2016). A person who merely says that some novel action is wrong, but does not care if others engage in that novel action or who herself engages in the action, cannot be said to have adopted the norm (Dahl \& Killen, 2018). Conversely, a person who follows the norm, but does not actually believe that engaging in the novel action is wrong, cannot be said to have adopted the new norm, either. A fully-developed normativity requires norms to guide our judgments, reasoning, emotions, and actions.

Traditionally, research in normative development has focused on the cognitive component of norm endorsement (e.g. Nucci \& Turiel, 1978; Smetana, 1985; Turiel, 1983; 
Zelazo et al., 1996). In these studies, children's normative understanding is assessed via interview questions that prompt children to reason explicitly about the norm in question, and children are asked to compare transgressions across multiple dimensions. In contrast, a more recent line of research on normative development - using the "spontaneous intervention" method - has focused more on the motivational elements of norm endorsement (e.g. Heyman et al., 2016; Rakoczy \& Schmidt, 2013; Rakoczy et al., 2008; Vaish et al., 2011). In spontaneous intervention studies, children are typically introduced to a novel norm and then observe a third-party agent violating this norm. Children's spontaneous interventions to these transgressions are taken as an indicator of their normative sensitivity. Children have been shown to spontaneously intervene in response to third-party violations of a range of different moral (e.g. Heyman et al., 2016; Vaish et al., 2011; Yucel \& Vaish, 2018) and conventional norms (e.g. Rakoczy, 2008; Rakoczy \& Tomasello, 2009; Rakoczy et al., 2008; Wyman et al., 2009).

A few studies have used the intervention paradigm to compare children's interventions in moral and conventional situations, indicating that children distinguish between moral and conventional situations in their spontaneous interventions (Hardecker et al., 2016; Josephs et al., 2016; Schmidt et al., 2012). However, research that systematically integrates the cognitive and the motivational components of norm endorsement is still lacking. Therefore, the second goal of the research presented here was to employ both explicit interview questions as well as behavioral measures assessing the motivational elements of norm endorsement - such as participants' spontaneous interventions during norm transgressions, and participants' own adherence to the norm - within the same paradigm. 


\section{How do Preschoolers' and Adults' Assumptions about a Transgressor's Knowledge}

\section{Affect their Normative Evaluations and Interventions?}

Finally, our third goal was to investigate how children and adults track a transgressor's knowledge of a norm and whether they respond differently to norm violations as a function of whether the transgressor knows the norm. Previous research has shown that both adults (see e.g. Giffin \& Lombrozo, 2015) and children (see e.g. Cushman et al., 2013; Killen, Mulvey, Richardson, Jampol, \& Woodward, 2011; Proft \& Rakoczy, 2019) usually judge a transgressor who violates a norm unintentionally and unknowingly more leniently than someone who violates that norm on purpose and with full knowledge of its consequences. In these previous studies, however, knowledge was usually manipulated such that the transgressor knew about the norm at stake, but had a false belief about whether her action violated the norm (e.g., Proft \& Rakoczy, 2019).

In contrast, we tested whether children and adults respond differently to third-party norm violations as a function of whether the transgressor has had certain critical experiences from which they could learn the norm. We reasoned that one does not have to be explicitly taught that many moral violations are wrong because their intrinsic consequences for victims can be observed. Thus, we expected that participants would attribute knowledge of a moral norm to a puppet who had merely observed that an action caused pain to a victim. In contrast, we reasoned that one does have to be taught that conventional violations are wrong, because conventional norms lack direct, harmful consequences to a victim. We therefore expected that participants would attribute knowledge of a conventional norm to a puppet only if the puppet had seen an authority figure prohibit the action.

\section{The present studies}

To address the three interrelated research objectives described above, we conducted two experiments. In both studies, we tested preschoolers and adults. Children in Experiment 
1 were between 3 and 5-years-old. Age 3 is the earliest age at which both research traditions discussed above - interview studies (Killen \& Smetana, 2015) and spontaneous intervention studies (e.g., Schmidt et al., 2012) - have found evidence for children making the moral/conventional distinction. However, prior research has also shown that from age 3 to age 5, children's ability to distinguish between moral and conventional situations becomes more nuanced and reliable (see Killen \& Smetana, 2015), which is why we focused on this age range for Experiment 1. In Experiment 2, we tested only 5-year-olds and adults, following up on some of the primary findings of Experiment 1.

In both experiments, participants watched a video in which a puppet carried out a novel action that was subsequently prohibited by an authority figure. Depending on condition, the situation involved either a novel moral or conventional violation. In the moral situations, the puppet pressed on a spot on a "victim" puppet's hand and the puppet said "ow". In the conventional situations, the puppet pressed on a button on a box and the box made a "beep" sound. Our goal in designing these experimental conditions was to define two minimally different situations; one containing characteristic features of moral situations (the harm of a victim), and the other one containing characteristic features of conventional situations (prohibition by an authority without inherent consequences). At first sight, it might seem more challenging for a researcher to construct a novel moral norm than to construct a novel conventional norm. While in many situations, it would be possible to decide on an alternative convention that would solve the underlying "coordination problem" equally well (Lewis, 1969; Turiel, 1983), moral norms are less arbitrary by definition. However, moral norms about physical force can be highly specific, as well (Dahl \& Freda, 2017). When children begin to throw a ball, for instance, they will experience that throwing a ball into another person's face will elicit pain and prohibitions, whereas throwing a ball into another person's hands will generally not. Thus, in their everyday lives, children encounter new and specific 
conventional and moral norms. Similarly, in our experiments, we presented children with live situations involving novel actions, and explored how they reasoned about and reacted to violations of these norms.

To simultaneously probe the cognitive and motivational dimensions of normativity, we asked participants for explicit judgments and justifications about the transgressions they observed, and also assessed a range of behavioral measures. In addition to manipulating the type of norm that was violated, we also systematically varied the puppet's observation status: participants watched the video in which the novel action was prohibited either together with the transgressor (so that it would be clear that the puppet knew the norm) or while the transgressor was absent (so that the puppet could not be expected to know the norm). The two factors were manipulated between subjects and crossed to create four experimental conditions.

Regarding our first research question, we predicted that children and adults would construct distinct moral and conventional norms in the moral and conventional conditions, as evidenced by their distinct reasons for why the norm violations were wrong, as well as by their judgments about the moral norms being more generalizable and less alterable. We also predicted that participants would judge the moral violations as more severe than the conventional violations. For our second research question, we hypothesized that participants would show norm endorsement not only in their verbal responses but also in their interventions against the norm violations and their refusal to violate the norms themselves. For our third research question, we predicted that when the puppet had watched the video in which the novel norm was introduced alongside the participant (observation conditions), participants would initially think that the puppet knew the norm. In contrast, when participants had watched the video by themselves (no observation conditions), they would infer that the puppet did not know the norm. However, we expected that after participants 
observed the puppet's live transgression, participants in the moral, no observation condition would be less likely to say that the puppet thinks the action is "okay" than participants in the conventional, no observation condition, since only in the moral case would the transgressor puppet have observed the victim's pain signal and could thus have learned about the norm.

\section{Experiment 1}

\section{Methods}

\section{Participants}

A total of 144 children participated in three age groups: 48 3-year-olds $\left(M_{a g e}=3.6\right.$ years, $S D_{a g s}=0.2$ years, 26 female), 484 -year-olds ( $M_{a g e}=4.6$ years, $S D_{a g e}=0.3$ years, 24 female), and 485 -year-olds ( $M_{a s e}=5.5$ years, $S D_{a g e}=0.3$ years, 24 female). We tested 25 additional children but their data was excluded due to loss of the video data $(\mathrm{N}=12)$, child refusal or inability to follow experimenter instructions $(\mathrm{N}=9)$, or procedural errors $(\mathrm{N}=4)$. Children were recruited from a large metropolitan area in the Western United States, with a population of approximately 59\% white, 20\% Asian, 11\% Latinx, 8\% Black, and 7\% from two or more races. Children came from primarily middle- and upper-class families; their parents had volunteered to participate in child development studies. Children received a small gift for their participation.

The adult sample consisted of 48 undergraduate students $\left(M_{a_{8}}=20.4\right.$ years, $S D_{a g e}=2.0$ years, 24 female) who participated for course credit. Participants were recruited from a research participation pool at a large research university in the Western United States and compensated with course credit for their participation. At the time of data collection, the majority of undergraduate students at this university were Asian (35\%), followed by white (25\%), Latinx (15\%), then international students (12\%), students with two or more races $(6 \%)$, and Black students (2\%). 
The total sample size for Experiment 1 is comparable to that of previous studies (e.g., Proft \& Rakoczy, 2019; Killen et al., 2011). Data were collected between 2015 and 2017. All participants were tested in the lab and had English as their primary language. In each age group, 12 participants were assigned to each of the four conditions (moral, observation; moral, no observation; conventional, observation; conventional, no observation). All materials for Experiments 1 and 2, including data, analysis scripts, and stimulus videos are available here: https://osf.io/vd7hw/?view_only=9aa27f3fb37a43339d3378bcd091562e.

\section{Procedure}

Each test session was conducted by two experimenters. Experimenter 1 (E1) led the session, while experimenter 2 (E2) animated the different puppets.

Warm-up phase. The session started with a warm-up phase during which the participant was 1) familiarized with a smiley scale that would later be used to assess the participant's evaluation of the severity of the normative transgressions, 2) introduced to Max, the puppet with whom participants would interact throughout the game, 3) provided with some opportunities to protest against against Max' actions, and 4) introduced to the idea that Max could not hear anything when he was in his house, and thus would not hear the participant's evaluation of his actions (for details, see Supplementary Materials).

Video phase and manipulation of norm type. During the video phase, the participant - together with Max in the observation conditions - watched a video in which a puppet named Chris carried out a novel action. In the moral conditions, this action consisted of Chris causing pain to another puppet, Timmy, by pressing on a spot on Timmy's hand, whereupon Timmy exclaimed "ow". In the conventional conditions, Chris pressed a button on a box introduced as a "tima", causing a "beep" sound. After Chris had carried out the respective action, an authority figure, the "teacher", prohibited it by exclaiming "No! Don't press there." (in both the moral and conventional conditions). 
After the end of the video, E1 asked the participant a range of questions. First, E1 asked "Do you think Max saw the movie?" (puppet's observation status). Max was outside of his house while this question was asked and provided feedback on the participant's response. Then, Max went back into his house and E1 asked "Do you think Max thinks it's okay to press on [Timmy / the tima]?" and "why" or "why not" (attribution of evaluation to puppet and justification; justifications are reported and analyzed in Supplementary Materials) and "Do you think it's okay to press on [Timmy / the tima]?" and "why" or "why not" (own evaluation and justification).

Live phase. Next, E2 brought out Timmy or the tima (depending on condition) and E1 said "oh look, here is Timmy / the tima again,"; then turned away. Max looked over to Timmy / the tima and said "Oh, look! Watch what I'm going to do." He approached Timmy / the tima and pressed on him / it, then took a few steps back and turned his face to the participant. He said "Look! Watch this!" and pressed a second, and finally a third time.

Max left, and E1 turned back to the participant and asked "Do you think it was okay when Max kept on pressing on [Timmy / the tima]?" and "why" or "why not"” (participant evaluation of target action and justification). Next, E1 brought out the smiley scale introduced during the warm-up phase and asked "Can you show me how you liked it when Max kept pressing on [Timmy / the tima], and make [Timmy / the tima] make that sound?" (severity rating of target action). The scale ranged from ' 1 ', "really don't like" to ' 5 ', "really like". E1 then asked the following questions: 1) "do you think Max thought it was okay when Max pressed on [Timmy / the tima] again?" and "why" or "why not" (attribution of evaluation to puppet and justification), 2) "would it be okay for Max to press on [Timmy / the tima] if the teacher said it was okay to press on [Timmy / the tima] and make them make that sound?" and "why” or "why not" (alterability of rule by teacher and justification), 3) "do you think kids at other schools are allowed to press on [Timmy / the tima] and make 
them make that sound?" (generalizability of rule), and 4) "would you like to press on [Timmy / the tima]?" (own transgression). An overview of the experimental procedures for Experiments 1 and 2 is depicted in Figure 1. The experimental procedures for both studies were approved by the institutional review board of the university at which the research was conducted.

\section{Figure 1}

Experiments 1 and 2: Overview of the experimental procedures.

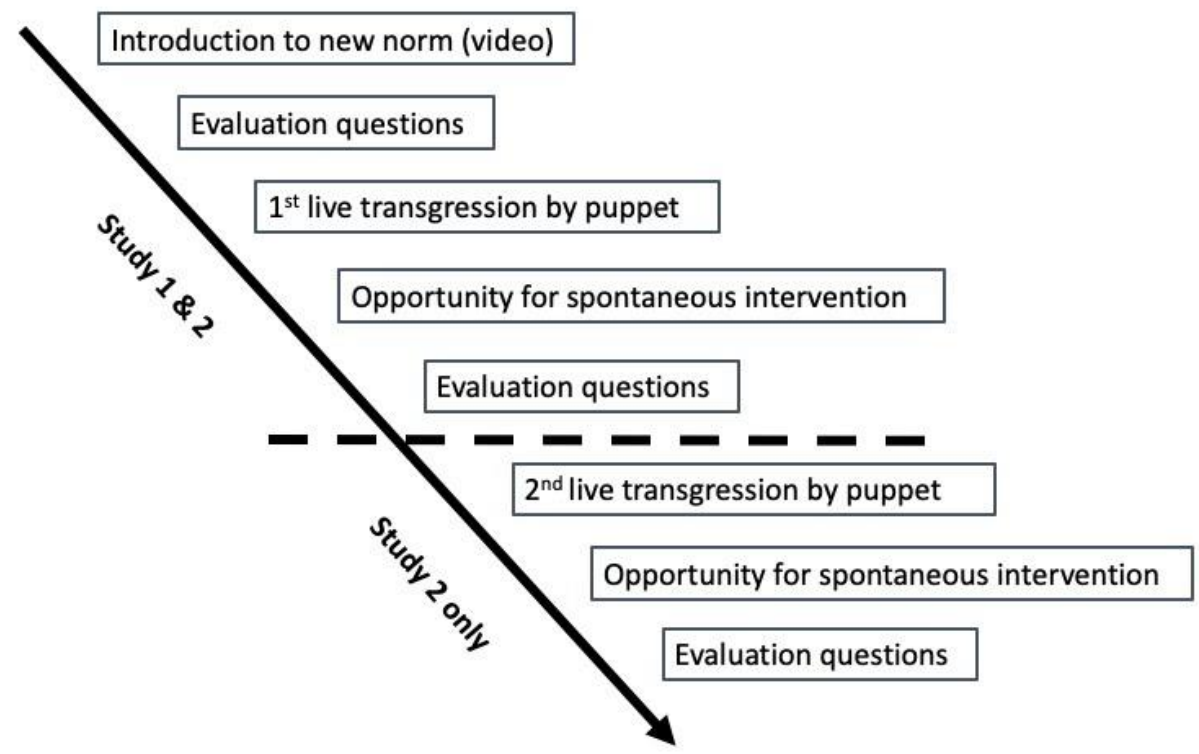

Note. In Experiment 1, the puppet transgressed the norm three times in a row and participants evaluated the puppet's behavior afterwards. In Experiment 2, the puppet transgressed the norm once, followed by a first evaluation phase; and then two more times, followed by a second evaluation phase.

\section{Coding}

Sessions were videotaped and coded by different researchers. All relevant verbal responses were first transcribed verbatim and then coded as described below; all relevant 
non-verbal responses were first qualitatively described and then coded as described below. A second coder coded $20 \%$ of trials to assess agreement.

Video and live phase: Spontaneous interventions. We coded whether the participant intervened when the puppet violated the norm in the live phase. Spontaneous interventions included 1) tattles (verbally informing E1 or - for children only - the parent of the puppet's actions, e.g. "He's pressing it."), 2) verbal protests (saying something to the puppet while he was committing the transgression, e.g., "No", "stop", "you shouldn't do that!"), and 3) physical interventions (for example reaching over to try to prevent the puppet from pressing). Coders reliably assessed whether a participant intervened (Cohen's kappa $=0.89)$.

Video and live phase: Questions after observing transgression. Answers for most of the questions that E1 asked the participant were coded as either "yes", "no", or "I don't know”. For justification questions (e.g., "why do you think it was not okay?”), responses were coded as one or several of the categories listed in table 1. Coders reliably assessed whether participants referenced considerations about other's welfare, conventional reasons, or the puppet's knowledge $\left(M_{\text {kappa }}=0.76\right.$, range: $\left.0.71-0.83\right)$. 


\section{Table 1}

Justification categories

\begin{tabular}{ll}
\hline \multicolumn{1}{c}{ Code } & \\
\hline None & None / participant does not know. \\
Welfare & Reference to the victim's pain, hurt, liking, or band-aid \\
& (e.g. "because it hurt"). \\
Conventional & Rule or convention statements, reference to authority, the video, a sound, \\
& conventional noise or alarm (e.g., "because the teacher said so"). \\
Knowledge & Reference to whether Max saw the video or Max's state of knowledge \\
& (e.g., "because Max didn't see the video"). \\
Evaluation & An evaluation of the transgression (e.g., "because that's not nice"). \\
Agent desire & Reference to agent's (i.e., Max's or participant's) desires or liking agent \\
& desire (e.g. "Max wanted to press it!"). \\
& Other (does not fall into any of the above categories or is unintelligible).
\end{tabular}

\section{Results}

\section{Data analysis}

All data for Experiments 1 and 2 were analyzed using the stats package in R. Unless otherwise noted, data were analyzed using Generalized Linear Models with binomial error distribution and logistic link function (Dobson \& Barnett, 2008). We used separate models for adults and children. The predictors were norm type (moral vs. conventional), observation status (observation vs. no observation), and (for children only) age group, as well as the 2- 
way-interactions between these predictors. Models also included gender as a predictor, although no gender effects were hypothesized. Gender effects were rare and inconsistent; they are reported in the Supplementary Materials. Hypotheses were tested using likelihood ratio tests. We only report statistics for analyses that were either hypothesized or significant. Additional tests and statistics for both Experiment 1 and 2 can be found in the Supplementary Materials. When analyzing participant evaluations and attribution of evaluations to the puppet, we contrasted "not okay" and other responses, pooling "other" (including "I don't know") with "okay" responses, since our main question was whether participants endorsed the norm that pressing was wrong or attributed such evaluations to the puppet. Since there were so few "other" responses across the four studies, the findings remained effectively unchanged when "other" responses were excluded. Results are reported in the order in which the measures were presented to participants during the experiment.

\section{Interventions during warm-up phase}

During the warm-up trials, $98 \%$ of adults and $99 \%$ of children intervened at least once without being prompted by the experimenter.

\section{Video phase}

Puppet's observation status. Participants were more likely to say that Max saw the video in the observation conditions ( $96 \%$ adults, $83 \%$ children) than in the no observation conditions ( $0 \%$ adults, $10 \%$ children), $D(1)=60.89, p<.001$ (adults), $D(1)=89.00, p<.001$ (children). Since Max provided the correct answer right after participants' made this judgment, telling participants whether he saw the video, even participants who wrongly indicated the puppet's observation status were included in the rest of the analyses (note also that removing these participants did not alter the pattern of findings).

Attribution of evaluation to puppet. Adults were more likely to say that Max thought the target action was wrong in the observation conditions (92\%) than in the no observation 
conditions $(12 \%), D(1)=34.89, p<.001$. In contrast, most children in all four conditions thought that the puppet viewed the act as wrong, regardless of whether or not the puppet had seen the video (Figure 2).

\section{Figure 2}

Experiment 1: Proportion of participants' "not ok" responses to the question "Do you think Max thinks it's okay to press on Timmy / the tima?" after the video phase; depending on the transgressor's observation status.

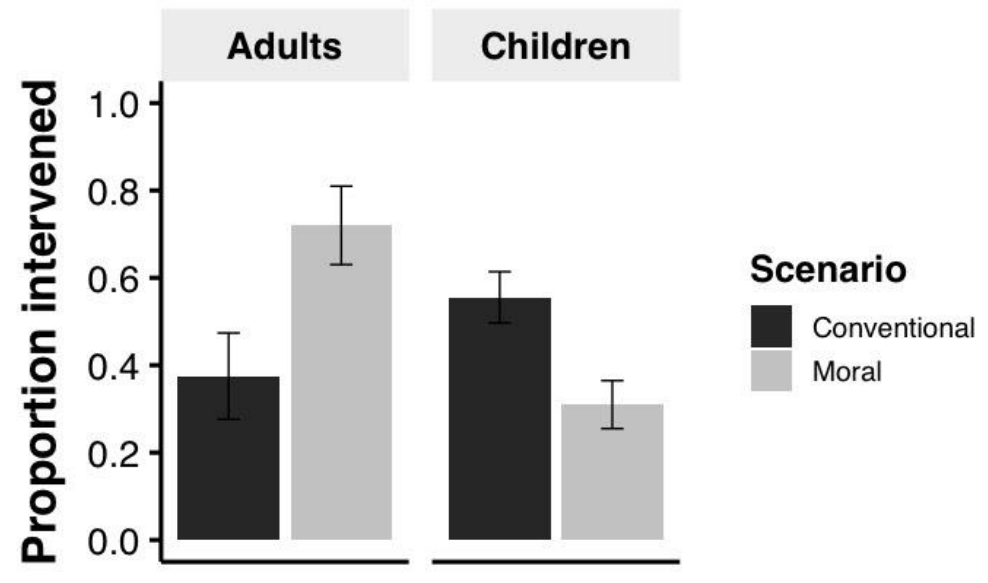

Note. The data indicate that children did not pick up on the observation manipulation, as children were not less likely to say that the transgressor lacked knowledge of the norm when he had not seen the video. Error bars indicate +/- $1 \mathrm{SE}$ of the population proportion.

Participant's own evaluation. Almost all adults (98\%) said the target action was wrong. When explaining why they thought the target action was wrong, adults used references to welfare exclusively in the moral conditions $(75 \%), D(1)=35.94, p<.001$. Conventional justifications were significantly more common in conventional conditions $(91 \%)$ than in moral conditions $(21 \%), D(1)=27.25, p<.001$. References to knowledge (11\%) were more common in the observation conditions $(22 \%)$ than in the no observation 
conditions $(0 \%), D(1)=7.84, p=.005$. Adults also referenced agent desire (2\%), and made evaluative statements $(2 \%)$.

Most children said the target action was wrong (78\%). 3-year-olds were more accepting of the target action (62\% not okay) than 4-year-olds (81\% not okay) and 5-yearolds (92\% not okay), $D(2)=13.82, p<.001$. Overall, children were more likely to say the target action was wrong in the moral conditions $(86 \%)$ than in the conventional conditions $(71 \%), D(1)=5.55, p=.019$, and - unexpectedly - more likely to say the target action was wrong in the no observation conditions (85\%) than in the observation conditions $(72 \%)$, $D(1)=4.04, p=.045$. When explaining why the action was wrong, children referenced welfare more often in the moral conditions $(68 \%)$ than in the conventional conditions $(2 \%)$, $D(1)=66.61, p<.001$, and conventional justifications more often in the conventional conditions $(63 \%)$ than in the moral conditions $(6 \%), D(1)=48.46, p<.001$. Conventional references were also more common in the observation conditions (37\%) than in the no observation conditions $(28 \%), D(1)=4.20, p=.041$. Children also mentioned agent desire (2\%), evaluation (3\%), and the puppet's knowledge (1\%).

\section{Live Phase}

Spontaneous interventions. Overall, adults intervened in 54\% and children in $44 \%$ of situations. Adults intervened more often in the moral conditions $(71 \%)$ than in the conventional conditions ( $38 \%), D(1)=5.70, p=.017$. Children intervened more in the conventional conditions $(56 \%)$ than in the moral conditions $(32 \%), D(1)=9.14, p=.002$. There was a significant interaction between age group and observation condition, $D(1)=7.26$, $p=.027: 3$-year-olds were more likely to intervene in the observation condition $(62 \%)$ than in the no observation condition $(21 \%), D(1)=17.23, p<.001$. There were no significant effects of observation status on interventions for 4-year-olds (50\% vs. 46\%) or 5-year-olds (38\% vs. 
$46 \%), p \mathrm{~s}>.53$ (Figure 3).

\section{Figure 3}

Experiment 1: Participants' spontaneous interventions separately for moral versus conventional scenarios.

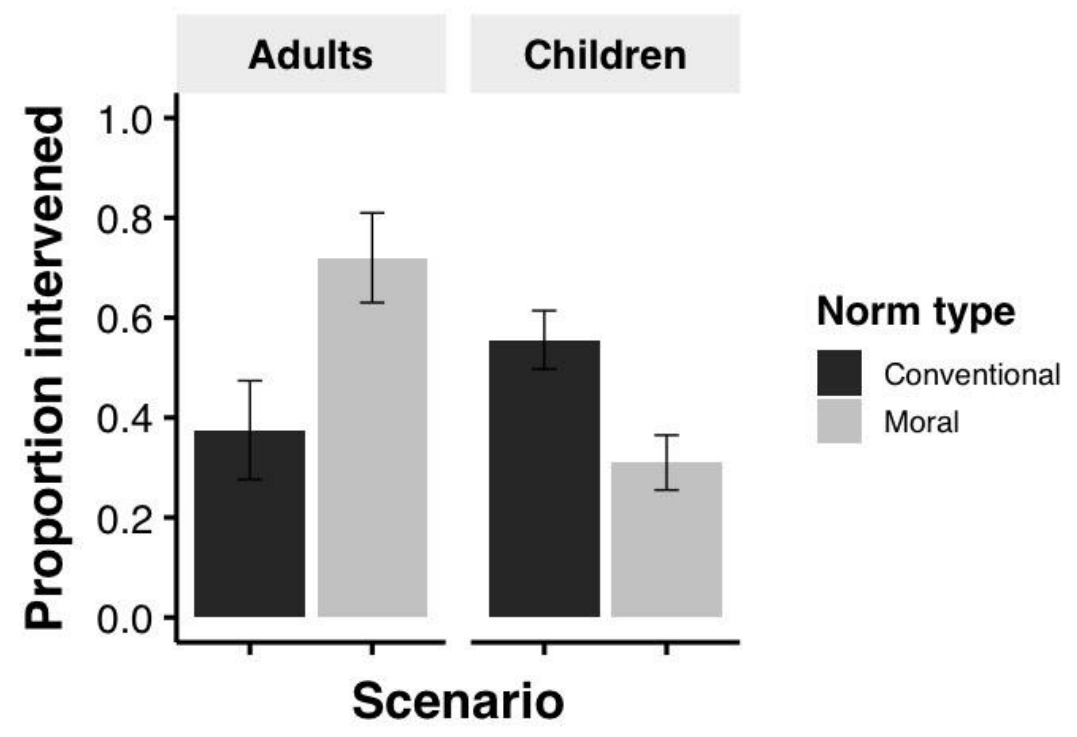

Note. Error bars indicate +/- $1 \mathrm{SE}$ of the population proportion.

Attribution of evaluation to puppet. After observing the live transgression, only $8 \%$ of adult participants thought the puppet viewed the target action as wrong. In children, 57\% said that the puppet thought the target action was wrong. Older children were less likely than younger children to say so (3-year-olds: 75\% not okay, 4-year-olds: 54\%, 5-year-olds: 42\%), $D(2)=11.68, p=.003$. Contrary to our predictions, there was no significant interaction between scenario type and observation status, $D(1)=1.93, p=.16$ (adults), $D(1)=0.53, p=.46$ (children).

Participant's own evaluation. Adults were more likely to say that the puppet's action was wrong in the moral conditions (100\%) than in the conventional conditions (79\%), $D(1)=7.83, p=.005$. When explaining why the puppet's action was wrong, adults referenced 
others' welfare exclusively in moral conditions $(96 \%), D(1)=53.33, p<.001$. Conventional justifications were more common in conventional conditions $(63 \%)$ than in moral conditions $(4 \%), D(1)=19.45, p<.001$. References to puppet knowledge were more common in the observation $(30 \%)$ than in the no observation conditions $(0 \%), D(1)=16.10, p<.001$, and more common in the conventional (26\%) than in the moral $(4 \%)$ conditions, $D(1)=10.46$, $p=.001$. Adults also made evaluative statements (5\%) and "other" statements $(2 \%)$.

Although most children in all age groups thought the puppet's action was wrong (87\%), older children were even more likely to say so (3-year-olds: $73 \%$ wrong, 4 -year-olds: 90\% wrong, 5-year-olds: $98 \%$ wrong), $D(2)=14.66, p<.001$. Among children who said that the puppet's action was wrong, references to others' welfare were more common in the moral situations $(86 \%)$ than in the conventional situations $(3 \%), D(1)=103.69, p<.001$, and conventional justifications were more common in the conventional scenarios (61\%) than in the moral scenarios $(6 \%), D(1)=47.33, p<.001$. The use of "other" statements was more common in the conventional situations $(7 \%)$ than in the moral ones $(0 \%), D(1)=5.81, p=.016$. Children used evaluative statements in $3 \%$ of trials and references to the puppet's knowledge in $2 \%$.

Severity rating of target action. Adults rated moral violations $(M=1.38)$ more severely than conventional violations $(M=2.33$; lower values indicate more negative evaluations), $F(1,43)=19.56, p<.001$. In children, there was a significant interaction between scenario and age group, $F(2,132)=4.01, p=.020: 3$-year-olds rated the moral transgression $(M=1.33)$ significantly more negatively than the conventional transgression $(M=3.00), t(45)=3.84, p<.001$, as did 4-year-olds (moral: $M=1.21$, conventional: $M=2.33$ ), $t(46)=3.38, p=.002$. In contrast, among 5-year-olds, the difference between ratings of moral $(M=1.29)$ and conventional $(M=1.58)$ events was not significant, $t(46)=1.23, p=.22$. 
Alterability of rule by teacher. When asked to imagine that the teacher had permitted the target action, most participants in the moral conditions said the action would still be wrong, even if the teacher gave permission ( $83 \%$ adults, $85 \%$ children). Only $4 \%$ of adults and $36 \%$ of children said it would still be wrong in the conventional conditions, $D(1)=37.48$, $p<.001$ (adults), $D(1)=38.78, p<.001$ (children).

Generalizability of rule. When asked whether it would be okay to engage in the target action at other schools, participants were significantly more likely to say the action would still be wrong in the moral conditions ( $96 \%$ adults, $94 \%$ children) than in the conventional conditions ( $75 \%$ adults, $79 \%$ children), $D(1)=4.61, p=.032$ (adults), $D(1)=8.06, p=.005$ (children). Within children, older children were overall more likely to say the action would still be wrong (3-year-olds: 75\%, 4-year-olds: 90\%, 5-year-olds: 96\%), $D(2)=10.55, p=.005$.

Participant's own transgression. In the conventional conditions, 50\% of adults engaged in the target action themselves. In the moral conditions, no adult decided to press, $D(1)=20.94, \mathrm{p}<.001$. Among children, $61 \%$ engaged in the target action in the conventional condition and $25 \%$ in the moral conditions, $D(1)=20.03, p<.001$ (see Figure 4 ). 


\section{Figure 4}

Experiment 1: Proportion of participants in the moral and the conventional scenarios who committed the respective normative transgression themselves.

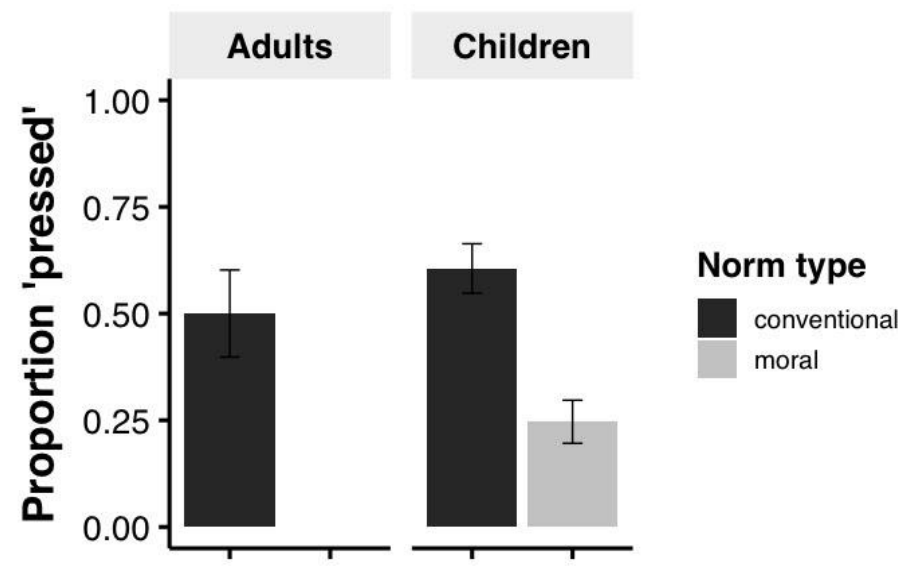

Note. Error bars indicate $+/-1 \mathrm{SE}$ of the population proportion.

\section{Discussion}

Both preschoolers and adults differentiated the novel moral and conventional situations on a variety of dimensions. When explaining why they thought the puppet's action was wrong, participants of both age groups were more likely to reference concerns about others' welfare in moral conditions and more likely to reference conventional reasons in the conventional conditions. Preschoolers and adults also considered the conventional violations as more alterable and less generalizable across contexts than the moral violation, and perceived the moral violations as more severe than the conventional ones. Thus, preschoolers, like adults, can rapidly construct distinct moral and conventional evaluations about novel actions based on distinct critical experiences associated with these actions, such as whether an action caused physical harm or was explicitly prohibited.

We also found that many adults and children in all conditions intervened to stop the transgressor puppet from violating the novel norm. Thus, we demonstrated both cognitive 
and motivational elements of norm endorsement within the same experiment. Interestingly, adults intervened more often in moral than in conventional situations, while children showed the reverse pattern. This suggests that different motivations may have been at work for adults versus children: adults may have predominantly intervened in order to prevent the harmful consequences of the transgressor's action from occurring. In contrast, one possible motivation behind children's interventions may have been to teach the transgressor about the norm at stake. This may have seemed particularly relevant with regard to the more arbitrary conventional norm. We will return to this point in the general discussion.

A second motivational element of norm endorsement that we assessed was whether participants were willing to engage in the normative transgression themselves. We found that participants of all ages were less likely to engage in the moral violation than in the conventional violation, demonstrating that some motivational elements of norm endorsement follow the same moral-conventional patterns as cognitive elements of norm endorsement.

Our third objective was to test whether assumptions about a transgressor's knowledge of a norm affect children's and adults' normative evaluations. Specifically, our question was whether, after the live transgression, participants would expect the transgressor in the no observation conditions to learn the moral norm - but not the conventional norm - by observing the direct negative consequences of their action (the victim's pain signal in the moral condition). Contrary to our predictions, adults and children in the moral, no observation condition were not more likely to say that the transgressor thought the action was wrong than adults and children in the conventional, no observation condition after observing the live transgression.

One possible explanation for this finding is that the transgressor's knowledge of the norm may have seemed ambiguous to participants. In particular, given that the puppet transgressed the norm three times without hesitation, participants may have inferred that the 
transgressor was aware of the norm but thought it was "okay" to violate it. This would be in line with previous research showing that people often draw inferences about an agent's intentions when they observe the agent violate a norm (e.g. Uttich \& Lombrozo, 2010) particularly when the agent does so repeatedly. We addressed this potential ambiguity of our knowledge manipulation in Experiment 2.

\section{Experiment 2}

Experiment 2 had three main goals. First, we wanted to replicate main findings from Experiment 1, regarding how preschoolers and adults rapidly construct distinct moral and conventional evaluations about novel actions.

Second, we expanded our assessment of participants' evaluations of the transgressor. To that end, we asked participants whether the transgressor should be punished, whether the transgressor was "mean," as opposed to "silly" (Dahl \& Schmidt, 2018; Nichols, 2002; Nucci \& Nucci, 1982; Smetana, 1981), and whether they thought the transgressor would engage in the prohibited action again after having engaged in it once.

Our third goal was to further investigate participants' normative expectations toward the transgressor puppet. In Experiment 1, the transgressor puppet violated the norm three times in a row. It was possible that participants assumed that the puppet could not think the act was wrong because the puppet kept doing it. In Experiment 2, therefore, the interviewer assessed participants' beliefs about whether or not the puppet knew the act was wrong after the first transgression, and then again after two further transgressions. Further, we tested only 5-year-olds (and not 3- and 4-year-olds) in Experiment 2 because some of our results in Experiment 1 had indicated that the younger children, in particular, struggled with understanding our observation manipulation. With these design changes, we were interested 
to see what participants believed that the puppet had learned from their first act of pressing on Timmy (moral) or the tima (conventional).

\section{Methods}

\section{Participants}

The child sample consisted of 485 -year-olds $\left(M_{\text {age }}=5.4\right.$ years, $S D_{\text {age }}=0.3$ years, 19 female). Data from 7 additional children was excluded due to technical problems $(\mathrm{N}=6)$, or because they responded to each of E1's questions with "I don't know" $(\mathrm{N}=1)$. The adult sample consisted of 80 undergraduate students ( $M_{a g e}=21.5$ years, $S D_{a g e}=3.5$ years, 40 female). Data from 4 additional adults was excluded due to technical problems $(\mathrm{N}=3)$ or experimenter error $(\mathrm{N}=1)$. Participants were recruited, compensated, and tested as in Experiment 1 and had similar demographic backgrounds.

Data collection for Experiment 2 started in 2018 and was interrupted in 2020 due to the COVID-19 pandemic. Participants were randomly assigned to the same four conditions as in Experiment 1. The target sample size for each age group in each of the four cells was 20. This sample size was determined based on simulations of statistical power, which yielded a power of approximately .9 for that sample size. Due to COVID-19, we were not able to reach the target sample size with children. 13 children each were assigned to the moral, observation and to the moral, no observation condition. 10 children were assigned to the conventional, observation condition and 12 children to the conventional, no observation condition.

\section{Procedure}

Warm-up and video phase. The warm-up and the video phase were the same as in Experiment 1, except that E1 provided participants with explicit feedback on the "Do you think Max thinks it's okay to press on [Timmy / the tima]?" question.

Live phase. Unlike in Experiment 1, Max violated the norm only once at first, followed by a first evaluation-phase. After that, Max transgressed the norm again twice, 
followed by a second round of evaluations (Figure 1). We changed some of the questions that E1 asked participants after observing the live transgressions. First, because Experiment 2 included an additional round of evaluations and we did not want to make the experiment too long overall, we omitted the questions regarding the authority-contingency and the contextdependency of the action, as well as the question about whether participants wanted to engage in the target action themselves. Second, to get a better understanding of participants' assessment of the consequences of the norm violations, E1 asked whether participants thought Max is "mean" or "just silly" (evaluation of mean vs. silly), and whether Max should get in trouble and "why" or "why not" (assignment of punishment). After Max' first live transgression, E1 also asked whether participants thought that Max would press again (prediction about repeated transgression).

\section{Coding}

The coding procedure was identical to Experiment 1. An independent coder coded $20 \%$ of the data Coders reliably reached agreement on whether a spontaneous intervention occurred (Cohen's kappa 0.84). Coders also reliably assessed whether participants referenced other's welfare, conventional considerations, or the puppet's knowledge $\left(M_{\text {kappa }}=0.81\right.$, range: $0.72-0.93)$.

\section{Results}

Because Experiment 2 involved a large number of assessments, in what follows, we will only report findings for a) measures that were newly introduced in Experiment 2, and that would help us address Experiment 2's second goal of expanding our assessment of participants' evaluations of the transgressor (evaluation of mean vs. silly; assignment of punishment; prediction about repeated transgression), and b) measures that addressed Experiment 2's third goal, tobetter understand how preschoolers' and adults' assumptions about a transgressor's knowledge of a new norm affect their normative evaluations and 
interventions (puppet's observation status; attribution of evaluation to puppet and justification). Additional results are reported in the Supplementary Materials (spontaneous interventions; participant evaluation of target action and justification; severity rating of target action).

\section{Video phase}

Puppet's observation status. Adults and children were far more likely to say that Max saw the target event in the observation conditions (adults 98\%; children 78\%) than in the no observation conditions (adults 0\%; children 8\%), Ds(1)=104.24 (adults) and 27.13 (children), $p s<.001$. As in Study 1, since Max provided the correct answer, even participants who wrongly indicated the puppet's observation status were included in the rest of the analyses. Removing these participants did not alter the pattern of findings.

Attribution of evaluation to puppet. Adults were more likely to say that Max thought the act was wrong in the observation conditions (92\%) than in the no observation conditions $(17 \%), D(1)=54.63, p<.001$. Unexpectedly, adults were also more likely to say that the puppet thought the act was wrong in the moral conditions $(62 \%)$ than in the conventional conditions $(48 \%), D(1)=4.50, p=.034$. As in Experiment 1, the majority of children in all four conditions said that Max thought the target action was wrong (moral observation; $92 \%$ not okay, moral no observation: $85 \%$ not okay, conventional observation: $80 \%$ not okay, conventional no observation: 67\% not okay, see Figure 5). Children's attributions did not significantly depend on any factors, $p s>.12$.

\section{First live phase}

Attribution of evaluation to puppet. After witnessing the puppet's first transgression, $31 \%$ of adults said that the puppet thought the target action was wrong. As predicted, adults showed a significant interaction between observation condition and scenario type, $D(1)=7.52, p=.006$ : in the observation conditions, there was no significant difference 
between the moral (25\%) and conventional (35\%) scenario, $D(1)=0.48, p=49$. In the no observation conditions, participants were significantly more likely to say that the puppet thought that the action was wrong in the moral scenario (55\%) than in the conventional scenario $(10 \%), D(1)=9.95, p=.001$. We also compared participants' attributions in the video phase versus the first live phase. In the moral scenarios, the difference in adults' attributions of negative judgments to the puppet did not differ (62\% "not okay" after the video phase versus $40 \%$ after the first live phase, $p=.12$ ). In contrast, in the conventional conditions, adults became less likely to think that the puppet thought the action was wrong from the video phase (48\%) to the first live phase (22\%), McNemar test: $p=.013$.

Among children, 56\% said that the puppet thought the target action was wrong. Children were more likely to say the puppet thought the action was wrong in the moral (73\%) than in the conventional conditions (36\%), $D(1)=10.21, p=.001$. There was no significant interaction between observation condition and scenario type; $D(1)=3.70, p=.054$. Comparing children's attributions in the video phase versus the first live phase, children's propensity to say that the puppet thought the action was wrong did not change significantly in the moral conditions ( $88 \%$ video phase, $73 \%$ first live phase, $p=.29$ ). Children in the conventional conditions became less likely to say that the puppet thought the action was wrong from the video phase $(73 \%)$ to the first live phase $(36 \%)$ than they were after the video phase, McNemar test: $p=.021$ (see Figure 5). 


\section{Figure 5}

Experiment 2: Participants' responses to the question "Do you think Max thinks it's okay to press on Timmy / the tima?" after the video phase, the first live phase, and the second live phase, respectively; depending on Max' knowledge status (observation versus no observation).

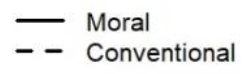

\section{Adults}

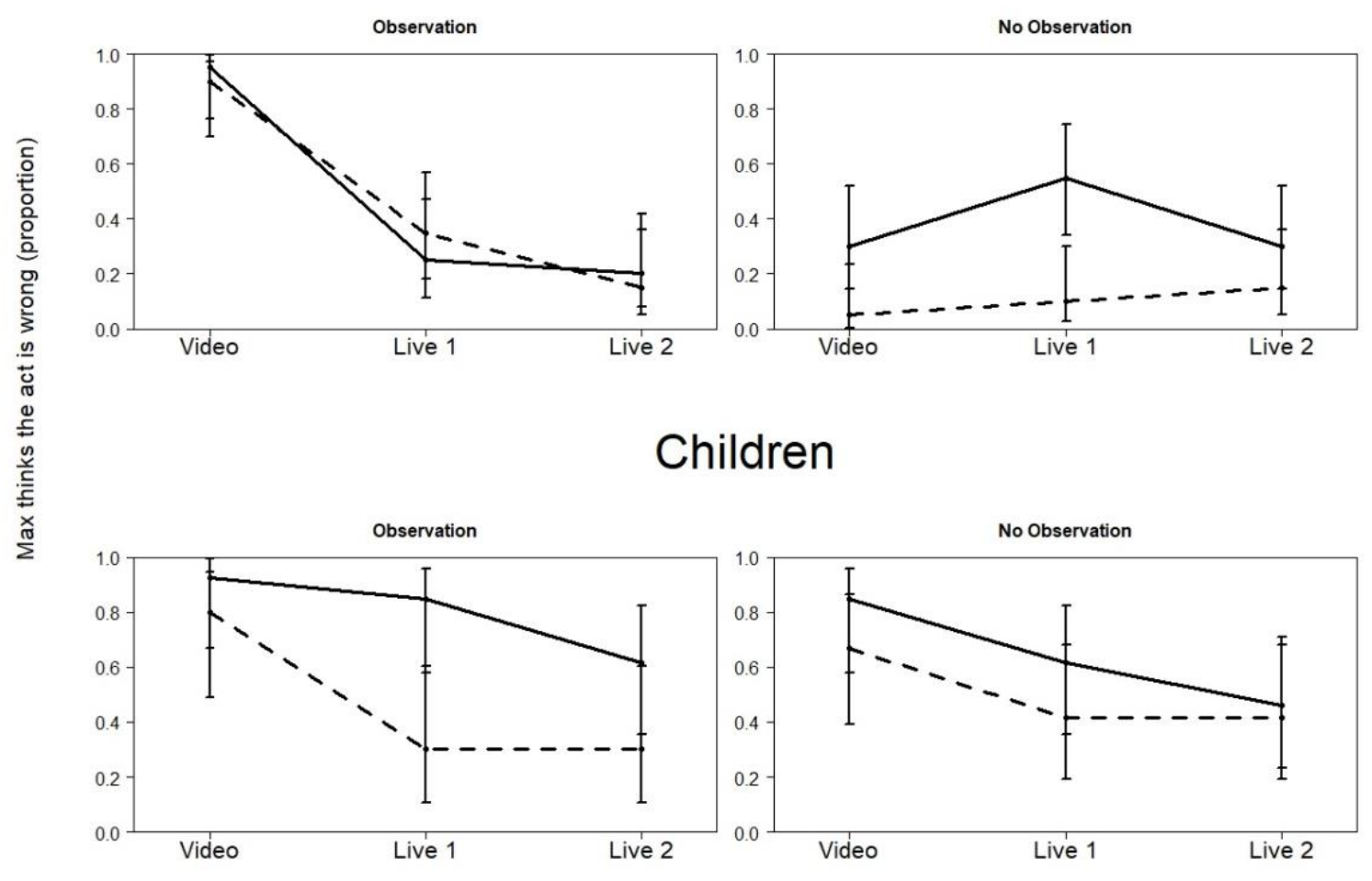

Note. Error bars indicate +/- $1 \mathrm{SE}$ of the population proportion.

Evaluation of means vs. silly. Participants were more likely to say that the puppet was "mean", rather than "silly", in the moral conditions (percentage saying "mean": 17\% adults, $46 \%$ children) than in the conventional conditions (percentage saying "mean": $0 \%$ adults, $14 \%$ children), $D(1)=10.82, p=.001$ (adults), $D(1)=5.68, p=.017$ (children). In adults, "mean" 
evaluations were also more common in the observation condition $(15 \%)$ than in the no observation condition $(3 \%), D(1)=4.74, p=.029$.

Assignment of punishment. Adults were more likely to say that the puppet should get in trouble in the moral scenarios $(60 \%)$ than in the conventional scenarios $(17 \%)$, $D(1)=18.36, p<.001$. Adults were also more likely to assign punishment in the observation conditions $(52 \%)$ than in the no observation conditions $(25 \%), D(1)=8.55, p=.003$. Overall, $58 \%$ of children said that the puppet should get in trouble, but children's assignment of punishment was not influenced by any factors $p s>.35$.

Prediction about repeated transgression. Adults were more likely to guess that the puppet would press again in the conventional conditions $(82 \%)$ than in the moral conditions $(60 \%), D(1)=5.89, p=.015$. They were also more likely to predict that the puppet would press again in the observation condition (88\%) than in the no observation condition (55\%), $D(1)=11.68, p<.001$. Children predicted that the puppet would press again in $63 \%$ of cases, but this was not influenced by any factors.

\section{Second Live Phase}

Attribution of evaluation to puppet. Among adults, 20\% said the puppet thought the target action was wrong; among children, 46\% said so. There were no significant effects, $p s>.06$. McNemar tests revealed no significant changes in participants' propensity to say that the puppet thought the action was wrong from the first to the second live phase, $p \mathrm{~s}>.21$ (see Figure 5).

Evaluation of means versus silly. Participants of both age groups were more likely to say that the puppet was "mean", rather than "silly", in moral conditions (percentage saying "mean": $68 \%$ adults, $62 \%$ children) than in conventional conditions (percentage saying "mean": $3 \%$ adults, $23 \%$ children), $D(1)=46.49, p<.001$ (adults), $D(1)=10.61, p=.001$ (children). 
Assignment of punishment. When asked whether the puppet should get in trouble, $63 \%$ of adults said "yes." Adults were more likely to say that the puppet should be punished in the moral conditions (98\%) than in the conventional conditions $(28 \%), D(1)=51.43$, $p<.001$. Among children, $67 \%$ believed that the puppet should get in trouble after the second live transgression. There were no significant effects $p s>.07$.

\section{Discussion}

Replicating key findings from Experiment 1, we showed again that preschoolers and adults construed the moral and the conventional situation as distinct, evaluating them differently in their judgments (e.g., regarding whether the action was wrong) and justifications (e.g. regarding why the action was wrong). Also as in Experiment 1, children were more likely to intervene in conventional than in moral situations. The analyses for these measures can be found in the Supplementary Materials. A new finding was that, after seeing the puppet transgress once, adults were more likely to predict that the puppet would press again in the conventional condition than in the moral condition. This result is also in line with the general conclusion that children and adults rapidly construe evaluations of novel events based on moral or conventional concerns. Both children and adults also evaluated the transgressor puppet differently as a function of whether he violated the moral vs.

conventional norm. Children and adults were more likely to judge the transgressor puppet to be "mean" (versus "silly") after the moral violation than after the conventional violation. Adults also judged the moral violation as more punishable than the conventional violation. Thus, participants perceive violators of novel moral and conventional norms similarly to violators of familiar moral and conventional norms (Nichols, 2002).

Our final goal in Experiment 2 was to investigate in more detail when people think that an agent knows about a novel norm and whether these assumptions influence participants' evaluations of the agent's normative transgression. As in Experiment 1, adults' 
assumptions about whether or not the puppet knew the target action was wrong were based on whether or not he had watched the video. Moreover, adults in Experiment 2 also updated their assumptions about whether the puppet thought the target action was wrong based on his experiences. The interaction effect between norm type and observation status after the first live transgression suggests that adults inferred that the puppet must have learned about the moral norm (but not about the conventional norm) from observing the moral norm's inherent consequences (the victim's pain signal). Further supporting this idea is the finding that in the conventional conditions - but not in the moral conditions - adults became less likely to think that the puppet thought the action was wrong from the video phase to the first live phase. Plausibly, upon observing the puppet's transgression during the first live phase, adults may have reasoned that if the puppet engages in the act, he thinks it is okay to do so. However, in the moral conditions, the puppet then received information that could lead him to think the action is wrong (in form of the victim's pain signal). In contrast, in the conventional conditions, the puppet received no such signal, perhaps leaving him to continue to believe that the act is all right.

In contrast to adults, children did not infer the perpetrator's knowledge based on whether or not he had watched the video. As in Experiment 1, most children in all conditions initially believed that the transgressor knew about the norm. However, in Experiment 2, E1 provided explicit feedback on participants' initial knowledge assessment. Our data suggest that this feedback may have helped children correct their assumptions about whether or not the puppet thought the target action was wrong and update these assumptions as a consequence of the puppet's experiences (see Proft \& Rakoczy, 2019 for a similar effect of experimenter feedback). Specifically, like adults, children in the conventional conditions (where the puppet did not receive information that could lead him to think the action is wrong) - but not in the moral conditions (where the puppet received such information in form 
of the victim's pain signal) - became less likely to think that the puppet thought the action was wrong from the video phase to the first live phase.

\section{General Discussion}

The present research addressed three interrelated questions about the construction and application of norms. With regard to our first question, we demonstrated across two experiments that preschoolers and adults used distinct social experiences to rapidly construe a novel norm as moral or conventional. When they observed a transgression that had intrinsic consequences - in this case, the victim's pain - participants construed the novel norm as moral. In contrast, when the transgression did not have intrinsic consequences to others' rights or welfare, participants construed the novel norm as conventional. This distinction was evident across a range of measures, including measures that have been employed in previous studies (e.g. justification questions, questions about the generalizability and alterability of norms), as well as novel measures (e.g., predictions about whether the puppet would transgress the norm again).

Our findings provide a direct test of the idea that the origins of children's knowledge about moral and conventional norms lies in their everyday life social experiences - an idea which has been proposed by much prior theorizing (Piaget, 1970; Smetana, 2013; Turiel, 1983, 2002, 2015; see also Tomasello, 2019). By presenting our participants with novel norms in the context of live situations, we were able to more closely than previous experimental studies (e.g. Helwig et al., 2001; Zelazo et al., 1996) mimic everyday life situations in which children and adults have to extrapolate the rules that govern behavior from rich and rapidly unfolding events (Dahl \& Turiel, 2019). Our findings complement early observational work (e.g. Dahl et al., 2014; Nucci \& Nucci, 1982; Nucci \& Turiel, 1987; Siegal \& Storey, 1985) in showing that despite these challenging circumstances, even 
preschoolers can construe normative transgressions as moral or conventional based on the distinct social experiences with which these norms are associated.

Regarding our second objective, we showed that preschoolers and adults spontaneously intervene against the very same normative transgression (motivational elements) that they subsequently judge as wrong, mean, or punishable (cognitive elements). This bridges two methodological paradigms that have typically been segregated in prior work (e.g., Killen \& Smetana, 2015; Tomasello, 2019). Interestingly, we found across both experiments that children intervened more often in conventional than in moral situations. One possible interpretation of these findings is that children's main motivation for intervening was to teach the puppet about the norm because conventional norms can less easily be inferred from observing the consequences of one's action than moral norms against physical harm (Köymen \& Tomasello, 2020). Clearly, however, alternative explanations of why children intervened more often in the conventional than in the moral conditions are possible. For example, children may have been hesitant to intervene in the moral situations because they did not want to appear to have had any hand in causing harm. More research is needed to test these and alternative interpretations of such differences in average intervention rates.

In response to our third research question, we found clear evidence that adults master the challenge of inferring and updating an agent's knowledge about a new norm based on the agent's experiences. Regarding children, our findings are more mixed. First, most children in both experiments initially said that the puppet thought that the target action was wrong, even if the puppet had not watched the movie in which the norm was introduced (Killen et al., 2011). One reason for preschoolers' difficulties with inferring whether the puppet thought the target action would be wrong based on whether or not he watched the video might have been the complexity of our manipulation, which required a number of inferential steps: children had to know that the puppet did not watch the movie, think about what relevant 
information was conveyed in the movie (the prohibition against pressing), and reason that the puppet must not have learned this.

While children struggled with inferring whether the puppet knew his action was wrong based on whether or not he had watched the video, one of our findings suggests that children in Experiment 2 did appropriately update their assumptions about the puppet's knowledge based on his experiences during the first live transgression. However, the clearest indicator that children took the puppet's experiences into account when updating their assumptions about what he knew and did not know would have been the predicted interaction effect between norm type and observation status after the first live transgression - it would have indicated that children think that one can learn about moral norms, but not about conventional norms, from observing the inherent consequences of one's actions. Unlike in adults, we did not find this interaction effect in children. One important thing to note is that we did not reach our target sample size in Experiment 2 due to the COVID-19 pandemic; thus, it is possible that we may have failed to detect an interaction effect between norm type and observation status on children's evaluation due to limited statistical power.

An alternative interesting possibility is that 5-year-old children actually struggle with understanding that someone else is lacking knowledge of a norm, even if they themselves only just learned about this norm. This would be in line with findings from one other study that manipulated a transgressor's knowledge of a new norm, and also failed to detect an effect of this manipulation on 5-year-old children's normative judgments (Samland \& Waldmann, 2016). Additional research on how children and adults infer whether others know or do not know a novel norm would help foster our understanding not only of children's developing normative abilities, but also of how these abilities interact with children's Theory of Mind. 


\section{Conclusion}

Our research shows that preschoolers, like adults, rapidly pick out the relevant information from different social situations and use this information to construe a novel norm as moral or conventional. To our knowledge, we are the first to demonstrate that preschoolers distinguish novel moral and conventional norms - not only in their judgments and justifications, but also with regard to motivational elements of norm endorsement. Together, our findings suggest that the observations and experiences children make in their everyday lives provide an important context for learning about different types of novel norms. Our results also point to a number of interesting further questions on children's developing abilities to integrate other's knowledge of a norm into their normative judgments.

\section{Funding}

This research did not receive any specific grant from funding agencies in the public, commercial, or not-for-profit sectors. 


\section{References}

Cushman, F. (2008). Crime and punishment: Distinguishing the roles of causal and intentional analyses in moral judgment. Cognition, 108 (2), 353-380. https://doi.org/10.1016/j.cognition.2008.03.006

Cushman, F., Sheketoff, R., Wharton, S., \& Carey, S. (2013). The development of intentbased moral judgment. Cognition, 127 (1), 6 21.https://doi.org/10.1016/j.cognition.2012.11.008

Dahl, A. (2017). Ecological commitments: Why developmental science needs naturalistic methods. Child Development Perspectives, 11 (2), 79-84. https://doi.org/10.1111/cdep.12217

Dahl, A., \& Freda, G. F. (2017). How young children come to view harming others as wrong: A developmental analysis.

Dahl, A., \& Killen, M. (2018). Moral reasoning: Theory and research in Developmental Science. Stevens' Handbook of Experimental Psychology and Cognitive Neuroscience, 4, 1-31. https://doi.org/10.1002/9781119170174.epcn410

Dahl, A., \& Kim, L. (2014). Why is it bad to make a mess? preschoolers' conceptions of pragmatic norms. Cognitive Development, 32, 12-22. https://doi.org/10.1016/j.cogdev.2014.05.004

Dahl, A., \& Turiel, E. (2019). Using naturalistic recordings to study children's social perceptions and evaluations. Developmental psychology, 55 (7), 1453. https://doi.org/10.1037/dev0000735

Dahl, A., \& Waltzer, T. (2020). Constraints on conventions: Resolving two puzzles of conventionality. Cognition, 196, 104125. https://doi.org/10.1016/j.cognition.2019.104152

Davidson, P., Turiel, E., \& Black, A. (1983). The effect of stimulus familiarity on the use of 
criteria and justifications in children's social reasoning. British Journal of Developmental Psychology, 1 (1), 49-65. https://doi.org/10.1111/j.2044835X.1983.tb00543.x

Diesendruck, G., \& Markson, L. (2001). Children's avoidance of lexical overlap: A pragmatic account. Developmental Psychology, 37 (5), 630-641. https://doi.org/10.1037/0012-1649.37.5.630

Dobson, A. J., \& Barnett, A. G. (2008). An introduction to generalized linear models. Chapman; Hall/CRC.

Giffin, C., \& Lombrozo, T. (2015). Mental states are more important in evaluating moral than conventional violations. Proceedings of the 37 th annual conference of the Cognitive Science Society.

Hardecker, S., Schmidt, M. F., Roden, M., \& Tomasello, M. (2016). Young children's behavioral and emotional responses to different social norm violations. Journal of Experimental Child Psychology, 150, 364-379. https://doi.org/10.1016/j.jecp.2016.06.012

Helwig, C. C., Zelazo, P. D., \& Wilson, M. (2001). Children's judgments of psychological harm in normal and noncanonical situations. Child Development, 72 (1), 66-81. https://doi.org/10.1111/1467-8624.00266

Heyman, G. D., Loke, I. C., \& Lee, K. (2016). Children spontaneously police adults’ transgressions. Journal of Experimental Child Psychology, 150, 155-164. https://doi.org/10.1016/j.jecp.2016.05.012

Killen, M., Mulvey, K. L., Richardson, C., Jampol, N., \& Woodward, A. (2011). The accidental transgressor: Morally-relevant theory of mind. Cognition, 119 (2), 197-215. https://doi.org/10.1016/j.cognition.2011.01.006

Killen, M., \& Turiel, E. (1991). Children's reasoning about interpersonal and moral conflicts. 
Child Development, 62 (3), 629-644. https://doi.org/10.2307/1131136

Killen, M., \& Smetana, J. G. (2015). Origins and development of morality. Handbook of child psychology and developmental science, 1-49. https://doi.org/10.1002/9781118963418.childpsy317

Knobe, J. (2003). Intentional action and side effects in ordinary language. Analysis, 63 (3), 190-194. https://doi.org/10.1111/1467-8284.00419

Kochanska, G., \& Aksan, N. (2006). Children's conscience and self-regulation. Journal of Personality, 74(6), 1587-1618. https://doi.org/10.1111/j.1467-6494.2006.00421.x.

Köymen, B., \& Tomasello, M. (2020). The early ontogeny of reason giving. Child Development Perspectives, 14(4), 215-220.

Lewis, D. (1969). Convention: A philosophical study. John Wiley \& Sons.

Nichols, S. (2002). Norms with feeling: Towards a psychological account of moral judgment. Cognition, 84 (2), 221-236. https://doi.org/10.1016/S0010-0277(02)000483

Nucci, \& Nucci, M. S. (1982). Children's responses to moral and social conventional transgressions in free-play settings. Child Development, 1337-1342. https://doi.org/10.2307/1129024

Nucci, \& Turiel, E. (1978). Social interactions and the development of social concepts in preschool children. Child development, 400-407. https://doi.org/10.2307/1128704

Piaget, J. (1970). Science of education and the psychology of the child. Trans. D. Coltman.

Proft, M., \& Rakoczy, H. (2019). The ontogeny of intent-based normative judgments. Developmental Science, 22 (2), e12728. https://doi.org/10.1111/desc.12728

Rakoczy, H. (2008). Taking fiction seriously: Young children understand the normative structure of joint pretence games. Developmental Psychology, 44 (4), 1195. https://doi.org/10.1037/0012-1649.44.4.1195 
Rakoczy, H., \& Schmidt, M. F. (2013). The early ontogeny of social norms. Child Development Perspectives, 7 (1), 17-21. https://doi.org/10.1111/cdep.12010

Rakoczy, H., \& Tomasello, M. (2009). Done wrong or said wrong? young children understand the normative directions of fit of different speech acts. Cognition, 113 (2), 205-212. https://doi.org/10.1016/j.cognition.2009.07.013

Rakoczy, H., Warneken, F., \& Tomasello, M. (2008). The sources of normativity: Young children's awareness of the normative structure of games. Developmental Psychology, 44 (3), 875. https://doi.org/10.1037/0012-1649.44.3.875

Schmidt, M. F., Rakoczy, H., \& Tomasello, M. (2011). Young children attribute normativity to novel actions without pedagogy or normative language. Developmental Science, 14 (3), 530-539. https://doi.org/10.1111/j.1467-7687.2010.01000.x

Schmidt, M. F., Rakoczy, H., \& Tomasello, M. (2012). Young children enforce social norms selectively depending on the violator's group affiliation. Cognition, 124 (3), 325-333. https://doi.org/10.1016/j.cognition.2012.06.004

Siegal, M., \& Storey, R. M. (1985). Day care and children's conceptions of moral and social rules. Child Development, 1001-1008. https://doi.org/10.2307/1130111

Smetana, J. G. (1981). Preschool children's conceptions of moral and social rules. Child Development, 1333-1336. https://doi.org/10.2307/1129527

Smetana, J. G. (1985). Preschool children's conceptions of transgressions: Effects of varying moral and conventional domain-related attributes. Developmental Psychology, 21 (1), 18. https://doi.org/10.1037/0012-1649.21.1.18

Smetana, J. G. (2013). Moral development: The social domain theory view. In P. Zelazo (Ed.), Oxford handbook of developmental psychology (Vol. 1, pp. 832-866). New York, NY: Oxford University Press.

Smetana, J. G., \& Braeges, J. L. (1990). The development of toddlers' moral and 
conventional judgments. Merrill-Palmer Quarterly (1982-), 329-346. Smetana, J. G.,

Srinivasan, M., Foushee, R., Bartnof, A., \& Barner, D. (2019). Linguistic conventionality and the role of epistemic reasoning in children's mutual exclusivity inferences. Cognition, 189, 193-208. https://doi.org/10.1016/j.cognition.2019.04.001

Tisak, M. S., \& Turiel, E. (1988). Variation in seriousness of transgressions and children's moral and conventional concepts. Developmental Psychology, 24 (3), 352. https://doi.org/10.1037/0012-1649.24.3.352

Tomasello, M. (2016). A natural history of human morality. Harvard University Press.

Tomasello, M. (2019). Becoming human: A theory of ontogeny. Belknap Press.

Turiel, E. (1983). The development of social knowledge: Morality and convention. Cambridge University Press.

Turiel, E. (2002). The culture of morality: Social development, context, and conflict. Cambridge University Press. https://doi.org/10.1017/CBO9780511613500

Turiel, E. (2015). Moral development. Handbook of child psychology and developmental science, 1-39.

Turiel, E., \& Dahl, A. (2019). The development of domains of moral and conventional norms, coordination in decision-making, and the implications of social opposition. In The Normative Animal? (pp. 195-213). Oxford University Press.

Turiel, E., Killen, M., \& Helwig, C. C. (1987). Morality: Its structure, functions, and vagaries. The emergence of morality in young children, 155-243.

Uttich, K., \& Lombrozo, T. (2010). Norms inform mental state ascriptions: A rational explanation for the side-effect effect. Cognition, 116 (1), 87-100. https://doi.org/10.1016/j.cognition.2010.04.003

Vaish, A., Missana, M., \& Tomasello, M. (2011). Three-year-old children intervene in third- 
party moral transgressions. British Journal of Developmental Psychology, 29 (1), 124130. https://doi.org/10.1348/026151010X532888

Wyman, E., Rakoczy, H., \& Tomasello, M. (2009). Normativity and context in young children's pretend play. Cognitive Development, 24 (2), 146-155. https://doi.org/10.1016/j.cogdev.2009.01.003

Yucel, M., \& Vaish, A. (2018). Young children tattle to enforce moral norms. Social Development, 27 (4), 924-936. https://doi.org/10.1111/sode.12290

Zelazo, P. D., Helwig, C. C., \& Lau, A. (1996). Intention, act, and outcome in behavioral prediction and moral judgment. Child Development, 67 (5), 2478-2492. https://doi.org/10.2307/1131635 